\title{
Update on Research and Activities at the Centers for Disease Control and Prevention, and the Agency for Toxic Substances and Disease Registry
}

\author{
Richard Kleiman, MDa , E. Danielle Rentz, PhD ${ }^{b}$, Eyasu Teshale, MDc, Nicola Thompson, PhDc, \\ Helen Schurz-Rogers ${ }^{b}$
}

aDivision of Toxicology and Environmental Medicine, Agency for Toxic Substances and Disease Registry (ATSDR)

bHealth Studies Branch, Division of Environmental Hazards and Health Effects, National Center for Environmental Health (NCEH)

cEpidemiology and Surveillance Branch, Division of Viral Hepatitis, National Center for HIV, Viral Hepatitis, Sexually Transmitted

Diseases and Tuberculosis Prevention

\section{HEALTH STUDIES BRANCH (NCEH, CDC)}

The Health Studies Branch is responsible for responding to domestic and international requests for epidemiological assistance with suspected and known environmental-associated public health threats. These deployments are commonly referred to as EPI-AIDs. Over the last several years, scientists from the branch have responded to a variety of different chemical-associated public health threats. Examples include outbreaks of aflatoxinassociated illness in Africa [1], suspected ricin contamination of a United States postal facility in South Carolina [2], severe renal and neurological illnesses that were ultimately determined to be the result of diethylene glycol-contaminated cough syrup in the Republic of Panama [3], and others. The branch employs personnel with a wide variety of educational backgrounds and professional training, including epidemiology and different specialties and subspecialties of medicine, statistics, and other environmental health-related disciplines.

\section{Outbreak of Unknown Illness in Tigray Region of Ethopia (2007)}

During 2005, public health investigations by the regional Ethiopian health bureau and the national Ethiopian Ministry of Health (EMOH) identified 19 cases of an unusual illness. The illness was characterized by a diagnosis of chronic liver disease, which was preceded by mild symptoms of fever, weakness, and jaundice for about a year. This sickness was affecting members of a small, remote village in northern Ethiopia. Although no actual etiology was identified, it was ultimately determined that the outbreak was most likely associated with a toxin from a weed growing in the village's primary water source, a communal well. On the basis of this determination, the government relocated the entire village (350 households and 1500 people) and dismantled the well in January 2006. There were no additional reported cases of illness until the spring and summer of 2007, when numerous deaths and a number of new cases were identified in the resettled community. Upon the reappearance of persons with a similar pattern of illness, EMOH officials contacted CDC with a formal request for technical assistance. A multidisciplinary team was assembled and deployed to Ethiopia.

The team was comprised of 2 epidemiologists, an internal medicine physician, a health scientist, and a medical toxicology fellow. The overall investigation consisted of medical chart reviews to establish a case definition; a door-to-door census to enumerate the population and find additional cases; a case-control study to collect data through a questionnaire, a physical examination, and biological (blood and urine) samples; and environmental sampling of local plants, food, and traditional medicines. The medical toxicology fellow served as an on-site subject matter expert in chemical-associated illness as well as an integral member of the

Notes: The following is an update on research and activities in which medical toxicologists are actively involved at the National Center for Environmental Health and the Agency for Toxic Substances and Disease Registry (CDC/ATSDR). The Journal of Medical Toxicology will highlight periodically some of these activities to illustrate the growing relationship between medical toxicology and public health.

Editor's Note: The findings and conclusions in this article are those of the authors and do not necessarily represent the views of the Centers for Disease Control and Prevention or the Agency for Toxic Substances and Disease Registry. 
epidemiological and clinical investigation. During the clinical assessment of case patients, extensive splenomegaly was noted in several cases, facilitating further refinement of the differential diagnosis and case definition. The differential diagnosis for this outbreak was wide and included such agents as pyrrolizidine alkaloids (PAs), schistosomiasis, tropical splenomegaly syndrome, malaria, and others. Although there has been no conclusively identified etiology to date, this serves as a good opportunity to discuss PA toxicity.

\section{Comments}

The final cause of the outbreak in Ethiopia has yet to be determined, but the reports received from earlier investigations implicated an agent capable of producing hepatotoxicity. The morphology of the liver injury pattern can often be extremely helpful in identifying the responsible toxic agent, because different toxins are associated with different types of liver pathology. For example, acute hepatocellular necrosis is more consistent with arsenic or iron toxicity, while aflatoxin poisoning is more likely to result in microvesicular steatosis [4]. During the course of the original investigation into the first outbreak of illness in 2005, 2 postmortem liver biopsies were obtained from 2 separate patients presumed to have the disease in question. A local pathologist determined that the pattern of injury on 1 specimen was consistent with veno-occlusive disease (the other was not interpretable due to improper preparation). In this commentary, we present a discussion of potential etiological agents associated with veno-occlusive liver disease, with a focus on PAs. The reader is reminded that the final etiology has not yet been determined for this outbreak and that the authors used this incident as an opportunity to simply present a detailed review on one of the topic areas that arose.

\section{Background}

Veno-occlusive disease can be caused by a number of different agents, including cyclophosphamide, comfrey tea, and other alkaloid-containing plant preparations. PAs make up a large percentage of these plant alkaloids, and they usually impart a bitter taste upon mastication, a taste that is protective for the plant against herbivores. There have been almost 400 PAs identified in over 6,000 plants within numerous plant families to date. Most toxicity seems to result from three main families of plants: Boraginaceae, Compositae, and Leguminosae (Table 1) [5]. Multiple PAs have been found to possess a wide variety of adverse effects that can range in severity, depending on the agent and the dose of the exposure. Plants containing PAs can be found worldwide, and ingestional exposure can have serious implications for both humans and animals.

\section{Prevalence}

The true epidemiology of PA poisoning is difficult to establish, especially in areas with populations at risk of higher exposure, such as in Ethiopia and other parts of Africa. PAs have been implicated as the etiologic agent in a number of both animal and human mass poisonings in a variety of different countries [5]. In

\section{Table 1: Pyrrolizidine Alkaloid Containing Plants}

\begin{tabular}{lll}
\hline Family & \multicolumn{1}{c}{ Genus, species } & Common Name \\
\hline Boraginacaceae & Cynoglossum officinale & Hound's tongue \\
\hline Boraginacaceae & Amsinkia intermedia & tarweed \\
\hline Boraginacaceae & Heliotropium europaeum & heliotrope \\
\hline Boraginacaceae & Symphytum officinale & comfrey \\
\hline Compositae & Senecio cineraria & Dusty Miller \\
\hline Compositae & Senecio jacobaea & tansy ragwort \\
\hline Leguminosae & Crotalaria retusa & crotalaria \\
\hline
\end{tabular}

1974, there was an outbreak of PA-associated veno-occlusive disease in Afghanistan, where over 1,600 people became ill. This was later found to be secondary to ingestion of wheat contaminated with PA-containing Heliotropium seeds. There was a similar outbreak in India the following year in which nearly 30 people died from cereals mixed with PA-containing seeds from a plant in the Crotalaria family [6]. There have also been outbreaks of PAassociated human illness in other countries, including Jamaica and South Africa.

\section{Exposure}

The toxicity of the PAs depends on the specific plant and its type of toxin, the route of exposure, and the overall dose. Most PAassociated toxicity occurs after ingestion of PA-containing agents; however, it can also occur through dermal exposure. Absorption of these compounds after dermal application is low because of decreased bioavailability, but absorption can be increased if the compounds are applied to a skin barrier that is no longer intact, such as an abrasion or an area of inflammation. This increased absorption is relevant if these compounds are used to make topical local remedies used to treat areas without an intact skin barrier. There are no reported human accounts of dermal toxicity, but it continues to remain a theoretical concern [7]. However, ingestional exposure to PAs remains the primary exposure route that results in toxicity.

\section{Pathophysiology}

The liver typically plays an invaluable role in many aspects of normal physiology. The liver aids in the synthesis of several essential factors, the excretion of several metals and other compounds, and helps with the detoxification of several products of metabolism. The majority of the blood enters the liver through the portal vein, while the remainder enters as oxygenated blood for the liver through the hepatic artery. This blood then mixes in the sinusoids and travels through a meshwork before it eventually exits the liver through the terminal hepatic veins. This transport from the sinusoids to the central and terminal hepatic veins allows for oxygen delivery to the liver and clearance of byproducts; it also allows for enterohepatic circulation of some compounds. 
The pathophysiology of PA-induced veno-occlusive liver disease at a molecular level is complex. Various plants and PAs have different toxicities, but they are thought to work through the same mechanism. The alkaloids are metabolized by the cytochrome P450 system to N-oxides and conjugated dienic pyyroles. These $\mathrm{N}$-oxides can then be transformed into epoxides and toxic necines, but the main cause of injury appears to be through the pyyroles. The pyrroles affect structure and function of cells by reacting with proteins and nucleic acids $[7,8]$. The pyrroles are considered highly reactive, and they have the capability of producing 2 alkylating groups. Other agents with bifunctional alkylating agents, such as nitrogen mustards, are thought to alter mitosis by affecting cellular DNA [9]. Differences in hepatic metabolism among different species may explain why animals such as sheep and hamsters are resistant to PA toxicity. This understanding of the mechanism of PA toxicity not only offers a better understanding of the toxic mechanism of PA-containing plants, but it also hints at potential research avenues for future cancer treatments.

The toxicity of PAs is somewhat dependent on individual patient characteristics as well as the presence of medications or products that can alter PA metabolism. Since these compounds are metabolized by the cytochrome p450 system, they seem to be subject to the influence of other medicines or compounds that may alter this metabolism. Medications that induce the cytochrome p450 system (such as alcohol, corticosteroids, phenobarbitol, and St. John's Wort) result in increased metabolism of PAs, and the increase in production of metabolites results in an increase in toxicity. Other medications that inhibit the cytochrome p450 system (such as macrolide antibiotics) could potentially block or inhibit the formation of the pyrroles. There is also some genetic variation in the form of single-nucleotide polymorphisms, creating individual variability in the metabolism of these plant toxins $[7,8]$. This could potentially explain why certain populations within the same community are affected differently, even with similar exposures.

Pyrroles produced as a result of PA metabolism disrupt protein synthesis, as discussed above. Injury appears to occur mainly in the terminal hepatic veins and parenchyma, resulting in intimal layer thickening, with subsequent edema and eventually obstruction. Similar events may also occur in the central and sublobular hepatic veins; however, such events are more commonly found in the terminal venules themselves. On gross examination, the pathological liver specimen may show a "nutmeg" appearance, named because the centrilobular regions are red and brown and surrounded by the normal tan liver $[4,10]$. These structural changes create a mechanical obstruction and cause the subsequent veno-occlusive disease (also called sinusoidal obstruction syndrome) that is considered the pathological finding in PA toxicity. This obstructive process results in increased obstruction of portal blood flow through the hepatic veins and subsequent portal hypertension. There is also some evidence to suggest that damage to the endothelial cells and Kupffer liver cells results in decreased production of a prostaglandin that normally produces vasodilatory effects. These combined pathological effects result in the clinical manifestations, including hepatomegaly and ascites, of portal hypertension that may be seen $[7,8]$.

There have been lung injuries associated with PA toxicity, but such injuries have been seen only in animals. These lesions include pulmonary edema, hydrothorax, pulmonary congestion, and pulmonary arteritis. The underlying mechanism for this kind of injury is not yet defined, but it may be due to endothelial hyperplasia, mast-cell changes, changes in capillary permeability, or chronic spasm in the outflow portion of the pulmonary tract. While these pathological changes have not been reported to occur in humans, the changes are important considerations, since they have been found in animals and remain a theoretical concern for humans [9].

\section{Diagnosis and Presentation}

The diagnosis of PA toxicity in humans can be difficult for several reasons. First, outbreaks occur in remote areas of developing countries that may be medically underserved. Second, these same areas also have other parasitic diseases (schistosomiasis, leishmaniasis, tropical splenomegaly syndrome, malaria, and others) that may present similarly to PA toxicity [11-13]. There may also be social obstacles to overcome, since some of these exposures are due to traditional herbal remedies and are not readily accepted as harmful by community members. In addition, the community itself may be hesitant or just fail to reveal these treatments to health-care workers, thereby limiting the available history from which public-health investigators can glean information [14].

Patients may present with acute, subacute, or chronic findings, depending on the specific factors associated with their exposure. Clinical findings associated with toxicity include hepatomegaly, ascites, abdominal distension, collateral venous circulation, and hepatosplenomegaly. The insult to the hepatic veins results in the liver congestion noted above, and patients may develop findings consistent with liver disease. There seems to be some variability in the presentation, as some patients develop symptoms fairly quickly (within weeks), whereas others may have a slow chronic course that progresses for months to years [9]. Some sources report mortality as high as $40 \%$ for acute liver toxicity, but there are also reports of complete recovery in some patients [15]. People with a low-protein diet also seem to be more susceptible to PA toxicity and have a worse outcome. This is relevant because many of these areas where outbreaks occur often have populations suffering from malnutrition [9]. There is also some evidence that PA toxicity can be transferred through breast milk, and there has even been a case report of fetal demise with veno-occlusive disease in which the mother's diet was found to have high levels of PAs [16].

The diagnosis is often made through a proper history and a physical examination of the patient. When feasible, pathological biopsy findings consistent with veno-occlusive disease can confirm the diagnosis. It is difficult to test for PAs because there are hundreds of different toxic alkaloids. There are some reports of colorimetry and gas chromatography/mass spectrometry used to identify alkaloids in specific home remedies. These screening tests were later confirmed by observing toxicity of herbal extracts on 
hepatocytes in an in vitro cell culture [14]. Limitations of this type of testing include the fact that labs that can run these tests are not widely available, and that it is necessary to know what plant or other source should be investigated.

While the availability of this testing is limited, when such testing is available, it provides the potential to identify toxins early and allow for intervention. The most immediate intervention is cessation of exposure. This may be as simple as educating people about the dangers of some traditional remedies, or it could also involve eliminating a certain food source from a population's diet. The latter could have larger implications in a community in which food is not widely available. There have been studies that have used N-acetyl-l-cysteine (NAC) to demonstrate the oxidative role in injury caused by PAs $[7,8]$. This could potentially play a role in treatment of PA toxicity, since it does appear to be partly due to an oxidative injury in the liver.

\section{CONCLUSION}

The true cause of this particular outbreak has yet to be determined, however, outbreaks of PA-associated toxicity are a global public health problem. These outbreaks have caused significant morbidity and mortality in both animals and humans. It is important to consider PA toxicity in the differential for liver disease of unknown origin, and it is probably more important to do so in developing countries. A diagnosis of PA toxicity is mainly accomplished through a proper history and a physical examination of the affected patient. Specialized laboratory and liver biopsy testing, if available, may be helpful in confirming PA-associated toxicity. Local experts in native flora and vegetation should be involved in public health investigations into outbreaks of illness such as these, as they may be able to provide crucial information about specific plants to which a population is exposed. Once PA toxicity is suspected or confirmed, it is important to educate those persons with exposures about the inherent dangers associated with consumption of such plants. More research is needed on possible treatment options for PA-associated hepatotoxicity.

\section{REFERENCES}

1. Lewis L, Onsongo M, Njapau $\mathrm{H}$, et al. Aflatoxin contamination of commercial maize products during an outbreak of acute aflatoxicosis in Eastern and Central Kenya. Environ Health Perspect 2005;113(12):1763-1767.
2. Schier JG, Patel MM, Belson MG, Patel A, Schwartz M, Fitzpatrick N, et al. Public health investigation after the discovery of ricin in a South Carolina postal facility. Am J Public Health 2007;97 Suppl 1:S152-S157.

3. Schier JG, Algren A. Medical toxicology and public health: update on research and activities at the Centers for Disease Control and Prevention and the Agency For Toxic Substances and Disease Registry: outbreak of acute renal failure with neurological symptoms-Republic of Panama. J Med Toxicol 2007;2:85.

4. Flomenbaum NE, Goldman LR, Hoffman RS, et al. Goldfrank's Toxicologic Emergencies. 8th ed. New York: McGraw-Hill; 2006, 412-426.

5. Cheeke PR. Toxicity and Metabolism of Pyrrolizidine Alkaloids. J Anim Sci 1988;66:2343-2350.

6. Mohabbat O, Younos MS, Merzad AA, Srivastava RN, Sediq GG, Aram GN. An outbreak of hepatic veno-occlusive disease in North-Western Afghanistan. Lancet August 1976;2(7980)269-271.

7. Chojkier M. Hepatic sinusoidal-obstruction syndrome: toxicity of pyrrolizidine alkaloids. J Hepatol 2003;39:437-446.

8. Stewart MJ, Steenkamp V. Pyrrolizidine poisoning: a neglected area in human toxicology. Ther Drug Monit 2001;23:698-708.

9. McLean E. The toxic actions of pyrrolizidine (Senecio) alkaloids. Pharmacol Rev 1970;22(4):430-476.

10. Cotran RS, Kumar V, Collins T, editors. Robbins Pathologic Basis of Disease. 6th Edition. 1999;116-117.

11. Pande GK, Srinath CH, Pal S, Reddy KS. Hepatic venous outflow obstruction. Trop Gastroenterol 1998;19:82-95.

12. Haile T, Anderson SD. Visceral leishmaniasis in Northern Ethiopia. East Afr Med J 2006;83(7):389-392.

13. Abreha A, Habte-Gahr E. Observations of splenomegaly in Ethiopia. Ethiop Med J 1984;22;101-104.

14. Zuckerman M, Steenkamp V, Stewart MJ. Hepatic venoocclusive disease as a result of a traditional remedy: confirmation of toxic pyrrolizidine alkaloids as the cause, using an in vitro technique. J Clin Path 2002;55:676-679.

15. Shannon M, Borron S, Burns M, editors. Haddad and Winchester's Clinical Management of Poisoning and Drug Overdose. 4th edition. 2007;223-240.

16. Rasenack R, Müller C, Kleinschmidt M, Rasenack J, Wiedenfeld $\mathrm{H}$. Veno-occlusive disease in a fetus caused by pyrrolizidine alkaloids of food origin. Fetal Diagn Ther 2003;18(4):223-225. 